\title{
INDEX DE SÉLECTIONS ÉCONOMIQUES ET BIOLOGIQUES DES TAUREAUX SUR LA VALEUR BOUCHÈRE DE LEURS VEAUX
}

\author{
R. Rouvier, I. Gavrit,etr, B. Vissac. - Station de Génétique quantitative et appliquée, C.N.R.Z. \\ 78-Jouy-en-Josas
}

Dans la sélection sur descendance des taureaux sur la valeur de leurs veaux de boucherie, en France, les caractères à sélectionner sont le poids vif à 75 jours d'âge et la note de valeur bouchère de ces veaux. Le prix de vente au kilogramme (prix unitaire) augmente avec le poids vif à 75 jours et la note de valeur bouchère, ce qui indique l'importance économique de chacun de ces deux caractères. Il semble, de plus, que la valeur économique unitaire de chaque caractère soit fonction du niveau de ce caractère. Le véritable critère économique de sélection est le produit poids de vente $x$ prix unitaire, qui, compte tenu des valeurs des héritabilités et corrélations génétiques, peut être remplacé par le produit poids à 75 jours $x$ note de valeur bouchère. Il semble difficile d'obtenir un estimateur de la valeur génétique de ce produit, sauf dans l'hypothèse de log-normalité des distributions de ces deux caractères. Dans l'hypothèse de normalité de la distribution des valeurs brutes, nous discutons l'intérêt de certains index combinant additivement les deux caractères à sélectionner. Ces index sont établis dans le cas présent où la seule information que l'on ait sur l'objectif de la sélection est que celle-ci doit conduire à une augmentation des valeurs génétiques moyennes de chacun des deux caractères sélectionnés. Ces index I sont établis de façon à maximiser un progrès génétique global d'une combinaison linéaire $\mathbf{H}$ (appelée critère de sélection) des valeurs génétiques de ces deux caractères. Les coefficients de cette combinaison linéaire sont obtenus, sous une condition fixant la valeur de la somme de leurs carrés, de façon à maximiser l'écart-type $\sigma_{H}$ du critère de sélection, ou la corrélation $R_{H I}$ entre le critère et l'index, ou l'écart-type $\sigma_{I}$ de l'index. Cela conduit à maximiser d'une certaine façon l'espérance du progrès génétique de $\mathbf{H}$ qui s'écrit, en fonction de l'intensité de sélection $i$ :

$$
\mathrm{E}(\Delta \mathbf{H})=i \mathbf{R}_{H I} \sigma_{H}=i \sigma_{I}
$$

\section{III. - GÉNÉTIQUE QUANTITATIVE ET SÉLECTION}

\author{
ÉVOLUTION DES MODALITÉS DE RECRUTEMENT DES TAUREAUX \\ DESTINÉS A L'INSÉMINATION ARTIFICIELLE
}

J.-M. DuPLAN. - Chaire de Zootechnie, E.N.S.A., 78-Grignon

Le recrutement des taureaux de service destinés à l'insémination artificielle peut être réalisé de trois manières différentes qui constituent chacune un stade d'une même évolution : achat de vieux taureaux supposés connus d'après quelques descendants nés de monte naturelle, ou bien mise à l'épreuve de la descendance de taurillons en début de carrière reproductrice assez nombreuse pour qu'un tri puisse être opéré à la connaissance des résultats, ou encore achat de veaux mâles parfois produits sous contrat, passant en station de contrôle individuel de performances puis mis à l'épreuve comme les précédents. L'évolution a consisté, en France, en une réduction très marquée du premier mode, une apparition très limitée encore du troisième et un développement marqué du second. Ce développement est attesté par la diminution du rapport du nombre de vaches inséminées " $\mathrm{V}$ " au nombre de taurillons " $\mathrm{T}$ " mis à l'épreuve. Ce rapport est V/T passé 
de 33700 en 1958 à 10416 en 1968. Deux causes ont ajouté leurs effets : vulgarisation et désillusion entraînées par l'emploi de vieux taureaux mal connus; intense activité réglementaire des années 1964 et 1966. Il est intéressant de constater que, dans chaque cas, une progression marquée a eu lieu avant la sortie des textes. Le calcul d'un rapport V/T pour tout le pays ne rend pas compte des importantes disparités existant entre coopératives et entre races. C'est là où ce rapport est le plus bas que les potentialités d'amélioration sont les plus grandes. Il est vraisemblable, compte tenu des disponibilités en cheptel contrôlé et en moyens financiers, que le nombre de taurillons mis à l'épreuve ne dépassera guère à l'avenir 800 (toutes races, toutes productions). Les possibilités accrues d'utilisation offertes par le stockage de semence congelée doivent être consacrées à accroître la pression de sélection, plutôt qu'à réduire ce nombre. D'autre part, de nouveaux progrès peuvent être espérés, de la généralisation de l'achat de veaux mâles destinés à un premier tri sur performances individuelles.

\title{
TESTAGE DES TAUREAUX EN RACE MONTBÉLIARDE
}

\section{I. - CoMparaison ENTRE LES INDEX DES PARENTS ET DE LEURS Fir,}

\author{
G. RicordeAU, J. LEFEnvRE. - Station de Génétique quantitative et appliquée, \\ C.N.R.Z., 78-Jouy-en-Josas.
}

Cette étude concerne les données obtenues dans le cadre du testage des taureaux de race montbéliarde du Centre d'I.A. de Crançot dans le Jura. Les contrôles effectués de 1952 à 1962 portent sur la quantité de lait, le taux butyreux et le taux de protéines. Nous disposons au total de 52 couples mère-fils et de 24 couples père-fils.

La corrélation entre la valeur d'élevage des taureaux et l'index de leur mère (moyennes des lactations corrigées pour l'àge, niveau des premières lactations ou écart par rapport aux contemporaines de la même étable) est respectivement de $0,30,0,34$ et 0,37 , mais les régressions correspondantes sont faibles. Nous remarquons que l'intervalle de vêlage moyen des mères à taureaux est supérieur d'un mois à celui de l'ensemble de la population contrôlée (402 jours contre 373 en moyenne). Le coefficient de régression de l'index lait des fils sur l'index lait de leur père est seulement de 0,18 mais cela concerne uniquement 24 couples. Les coefficients de régression de l'index TB des fils sur l'index TB de leur mère ou de leur père sont respectivement de 0,182 et 0,483. Les coefficients de régression de l'index TP des fils sur l'index TP de leur mère ou de leur père sont respectivement de 0,176 et 0,285 . Par contre, les corrélations entre les index TB des parents et l'index TP des fils sont nulles; il en est pratiquement de même pour les corrélations entre l'index TP des parents et l'index TB de leurs fils.

La sélection indirecte sur le taux de protéines à partir du taux butyreux des parents semble donc inefficace. Ce résultat ne correspond pas avec la corrélation génétique entre le TB et le TP $(r=0,29)$ calculée par analyse de variance des lots de descendance.

\section{TESTAGE DES TAUREAUX EN RACE MONTBÉLIARDE}

\section{II. - Paramìtres GÉNÉtigues DE LA QUANTITÉ DE LAIT}

ET DE LA COMPOSITION DU IAIT EN MATIÈRE GRASSE, PROTÉINES ET CALCIUM

\author{
G. RICORDEAU, J. LEFEBVRE. - Station de Génétique quantitative et appliquée, \\ C.N.R.Z., 78-Jouy-en-Josas.
}

Ces données ont été obtenues dans le cadre du testage des taureaux de race montbéliarde du C.I.A. de Crançot de 1952 à 1962. Le contrôle fromager des filles de taureaux fournit la quantité de lait, le taux butyreux (TB), le taux de protéines (TP), et le taux de calcium. On a calculé ensuite le rapport TP/TB, la production journalière maximum et le coefficient de per- 